\section{The Genetics of Neuroendocrine Tumors: A Brief Overview}

Received: September 01, 2017; Accepted: September 09, 2017; Published: September 14, 2017

\section{Introduction}

Neuroendocrine cells were discovered in 1870 by Rudolph Heidenhain, who noted that they could lead to tumors. Siegfried Oberndorfer introduced the term carcinoid in 1907. Since that time numerous scientists have described tumors that secreted hormones causing a variety of symptoms. In 1924 Seale Harris described insulinoma, in 1942 William Becker described glucagonoma, and in 1955 Zollinger and Ellison described gastrinoma. The first reported case of CCK-oma was described in 2013. The term Neuroendocrine tumors (NET) encompasses a wide variety of neoplasms commonly secrete neuropeptides [1].

Multiple Endocrine Neoplasms (MEN) was first described by Underdahl et al. in 1953 however the term MEN was not coined till 1968 by Steiner et al. At that point, MEN syndrome was split into MEN 1 (Wermer syndrome) and MEN 2 (Sipple syndrome). men 2 was then subdivided into MEN 2A and MEN 3 in 1974. MEN 3 has been reclassified to MEN 2B and Familial Medullary Thyroid Cancer (MTC) has also been identified. Also, a new MEN syndrome (MEN 4) was described in 2006. Von-Hippel Lindau was originally described by Dr. von Hippel in 1904 and Dr. Lindau in 1927. Coined in 1936, the term Von-Hippel Lindau did not become common until the 1970's [2].

\section{Epidemiology}

The incidence of Neuroendocrine Tumors (NET) are increasing worldwide. In 1973, the incidence in the United States was 1.9 cases per 100,000 . In 2004, the incidence had increased to 5.25 per the SEER database. These same trends are occurring in numerous other countries [1].

While there has been no link between PPI use and NET, there is a temporal correlation between the increased use of proton pump inhibitors (PPI) and the increased incidence of NET. Timoprazole was discovered to inhibit acid secretion irrespective of stimulus in 1975. Further studies revealed enlargement of the thyroid gland due to reduced iodine uptake as well as atrophy of the thymus. Omeprazole began trials in 1980 and was released worldwide in 1988. It quickly became the biggest selling pharmaceutical in history. The long-term use of PPI has been shown to significantly increase gastrin levels. Gastrin stimulates neuroendocrine cell growth and may increase the malignant potential of NE cells [1].

\section{Matthew Helm ${ }^{1}$ and Saju Joseph ${ }^{2 *}$}

1 Texas Tech University Health Sciences Center, Lubbock Texas, USA

2 Graduate Medical Education Department, Valley Heath Systems Las Vegas Nevada, USA

\section{*Corresponding author: Saju Joseph \\ Saju.Joseph@uhsinc.com \\ Graduate Medical Education Department, Valley Heath Systems Las Vegas Nevada, USA.}

Citation: Helm M, Joseph S (2017) The Genetics of Neuroendocrine Tumors: A Brief Overview. J Clin Epigenet. Vol. 3 No. S2:33

\section{Genetics}

\section{MEN 1}

MEN 1 is associated with hyperparathyroidism, pituitary tumors, and pancreatic NET. Hyperparathyroidism occurs in $95 \%$ of patients, $20-40 \%$ will develop anterior pituitary lesions, and 40-80\% will develop pancreatic NET. Some MEN 1 patients will also develop adrenocortical tumors, lipomas, carcinoid tumors, angiofibromas, and collagenomas [1]. MEN 1 is associated with MEN1 gene, which encodes a 610 amino acid protein menin $[3,4]$.

Menin is a ubiquitously expressed nuclear protein in non-dividing cells. MEN 1 is located on chromosome 11 q13 and consists of 10 exons. Mutations in the MEN 1 gene causes inactivation of the menin protein and, $90 \%$ of tumors from MEN 1 patients have loss of heterzygosity (LOH). Thus, menin is believed to work as a tumor suppressor gene consistent with Knudson's "two-hit" hypothesis [2].

A MEN1 gene mutation is autosomal dominant and has a $90 \%$ penetrance by 40 years old. This inheritance pattern, along with the varying presentations of patient disease, correlates to the "two hit" hypothesis of oncogenesis. Furthermore, there have been over 1000 mutations recognized in MEN 1 patients. Most of these mutations lead to truncation of the menin protein and inactivation [5-14]. 
Menin has been shown to accumulate in the nucleus of most cells. The C-terminal quarter has at least 3 nuclear localization signals. In non-dividing cells menin is in the nucleus, while in dividing cells it is in the cytoplasm. Mutations of menin have been found to be missing at least one nuclear localization signal which may cause their accumulation in the cytoplasm. Menin appears to interact with several proteins involved in transcriptional regulation, genome stability, cell division and proliferation; however its exact function is still unknown. Menin has also been shown to directly bind double stranded DNA. This binding may play a role in epigenetic regulation via histone methylation or acetylation [6-15].

Despite the strong link between the MEN1 mutation and MEN 1 syndrome there remains $5-25 \%$ of MEN 1 patients who do not have an identified mutation of the MEN1 gene. This may be due to testing method differences that do not identify mutations that are present or include patients who have developed sporadic neoplasms without developing true MEN 1 syndrome. Finally, there is a $5-10 \%$ incidence of phenocopies in MEN 1 families [7-9,14].

\section{MEN 2}

MEN 2A causes medullary thyroid cancer, pheochromocytomas, and parathyroid adenomas. There is a complete penetrance for MTC, $50 \%$ incidence of pheochromocytomas, and $15-30 \%$ incidence of hyperparathyroidism. Most patients develop the MTC first, and a few can have a concomitant pheochromocytoma at their initial presentation. Unfortunately for MEN 2 patients pheochromocytomas are more likely to be bilateral, present at a younger age, and have less symptoms then patients with sporadic disease. Malignancy of the pheochromocytoma remains at a $4 \%$ risk. MEN 2B causes aggressive $\mathrm{MTC}$ at an early age and affecting all patients, again there is a $50 \%$ incidence of pheochromocytomas with half the patients having multiple lesions and bilateral disease. Parathyroid disease is absent in MEN 2B however most patients will have mucosal neuromas at a very early age with $40 \%$ developing GI ganglioneuromatosis. Finally, $75 \%$ of patients will have Marfanoid habitus and boney disorders $[5,16]$.

All MEN 2 cases are associated with the RET proto-oncogene which encodes a tyrosine kinase receptor. RET protein is a subunit of a multi-molecular complex that binds growth factors of the glial derived neurotropic factor family (GDNF). RET protein has an intracellular portion that contains 2 tyrosine kinase domains. The extracellular portion including 4 cadherin domains, a calciumbinding domain, and a cysteine-rich domain. There is also a transmembrane domain. Development of different subtypes of MEN 2 has been linked to different phenotypes and variable penetrance of the RET mutation $[10,15,16]$.

Patients with MEN 2A often have a gain of function mutation in the RET gene with the great majority mutating cysteine residues in exon 10 and 11 . Mutations in codon 634 in exon 11 has a high prevalence of pheochromocytoma and hyperparathyroidism $[6,15]$.

Patients with MEN 2B have been noted to have RET mutations as well with p.M918T most commonly associated with MEN 2B $[10,11,16]$.

\section{MEN 4}

MEN 4 is associated with and pituitary adenomas, pancreatic islet-cell hyperplasia, bilateral pheochromocytomas, paraganglionomas, and cataracts [2,12]. MEN 4 is mapped to CDNK1B gene mutation, which encodes the 195 amino acid cyclin dependent kinase inhibitor (CK1) p27 ${ }^{\mathrm{kip} 1}$. In a naturally occurring rat model, this syndrome, called MENX, is autosomal recessive. This CDNK1B mutation resulted in an absence of $\mathrm{p} 27$ protein in tumor cells. P27 belongs to the Cip/Kip family of cyclin dependent kinases. It binds and inhibits cyclin E-CDK2 which stops or slows cell cycle progression at G1. CDNK1B is located on chromosome $12 \mathrm{p} 13$. In humans, this mutation is autosomal dominant unlike MENX in rats $[2,12,13]$.

Von Hippel-Lindau: VHL patients develop both benign and malignant neoplasms like MEN patients. Classically they develop pheochromocytomas, paraganglionomas, and hemangioblastomas. VHL patients fall into 2 subtypes. Type 1 patients do not have pheochromocytomas while type 2 patients do.

VHL gene is a tumor suppressor gene on chromosome $3 p 25$. Approximately $20 \%$ of patients have large germline mutations, $27 \%$ have missense mutations, and $27 \%$ have nonsense or frameshift mutations. Finally, $20 \%$ of patients will not have an identifiable mutation on analysis. VHL protein binds elongin $B$ and $C$ and degrades the alpha subunit of hypoxia-inducible factor (HIF). Normal function of this protein seems to inhibit transcription elongation. Lack of normal alpha HIF degradation produces over stimulation of blood vessel growth and tumor development [14-17].

\section{Conclusions}

Neuroendocrine tumors are rare however the incidence is increasing. Identification of syndromes that lead to numerous different NET in the same patients has led to a better understanding of NET expression. Investigation of these syndromes has led to a better understanding of genetic mutations and the disease they are linked to.

1. MEN 1 syndrome is linked to the autosomal dominant MEN1 gene. The complete function of the menin protein is unknown currently, however inactivation has been shown to cause increased cell division and increased transcriptional mutations.

2. MEN 2 syndromes have been linked to the autosomal dominant RET proto-oncogene. RET codes for a tyrosine kinase receptor that binds GDNF and stimulates growth. RET mutations cause a gain in function that overstimulates growth. MEN 2 syndromes can be differentiated based on their RET mutation, phenotype variance and loss of heterzygosity.

3. MEN 4 is a newer syndrome associated with changes to the acid cyclin dependent kinase inhibitor and is mapped to mutations in the CDNK1B gene. This mutation causes loss of p27 protein which allows cells to continue to proliferate. It is autosomal dominant in humans.

4. VHL was previously not associated with NET however 
better understanding of its genetics has led to this connection. The $V H L$ gene mutations inhibit the normal degradation of alpha HIF allowing for transcriptional

\section{References}

1 Joseph S, Wang YZ, Boudreaux JP, Anthony LB, Campeau R, et al. (20110 Neuroendocrine Tumors: Current Recommendations for Diagnosis and Surgical Management. Endocrinol Metab Clin North Am 40: 205-231.

2 Thakker RV (2014) Multiple endocrine neoplasia type 1 (MEN1) and type 4(MEN4). Mol Cel Endocrinol 386: 2-15.

3 Chandrasekharappa SC, Guru SC, Manickam P, Olufemi SE, Collins FS, et al. (1997) Positional cloning of the gene for multiple endocrine neoplasia-type1. Science 276: 404-407.

4 Larsson C, Skogseid B, Oberg K, Nakamura Y, Nordenskjöld M (1988) Multiple endocrine neoplasia type 1 gene maps to chromosome 11 and is lost in insulinoma. Nature 332: 85-87.

5 Crona J, Skogseid B (2016) Genetics of neuroendocrine tumors. Eur J Endocrinol 174: R275-R290

6 Marx J (2005) Molecular genetics of multiple endocrine neoplasia types 1 and 2. Nat Rev Cancer 5: 367-375.

7 Öberg K (2013) The genetics of neuroendocrine tumors. Semin Oncol 40: $37-44$

8 Calender A (2000) Molecular genetics of neuroendocrine tumors. Digestion 62: 3-18. elongation and over stimulation of vascular growth. Hence, these patients often develop vascular tumors that can be either benign or malignant.

9 Heppner C (1997) Somatic mutation of the MEN1 gene in parathyroid tumors. Nature Gen 16: 375-378.

10 Moline J, Eng C (2011) Multiple endocrine neoplasia type 2: An overview. Genet Med 13: 755-764.

11 Alrezk R, Hannah-Shmouni F, Stratakis CA (2017) MEN4 and CDKN1B mutations: the latest of the MEN syndromes. Endocr Relat Cancer pii: ERC-17-0243.

12 Lee M, Pellegata NS (2013) Multiple endocrine neoplasia type 4. Front Horm Res 41 :63-78.

13 Pellegata NS (2012) MENX. Ann Endocrinol 73: 65-70.

14 Kaiwar C, Macklin SK, Gass JM, Jackson J, Klee EW, et al. (2017) Late onset pancreatic neuroendocrine tumor - a case report on the phenotypic expansion for MEN1. Hered Cancer Clin Pract 15: 10.

15 Norton JA, Krampitz G, Jensen RT (2015) Multiple endocrine neoplasia: genetics and clinical management. Surg Oncol Clin N Am 24:795-832.

16 Znaczko A, Donnelly DE, Morrison PJ (2014) Epidemiology, clinical features, and genetics of multiple endocrine neoplasia type $2 \mathrm{~B}$ in a complete population. Oncologist 19: 1284-1286.

17 Friedrich CA (1999) Von Hippel-Lindau Syndrome A pleomorphic condition. Cancer 86: 2478-2482. 